\title{
Sex Differences In Psychoeducation For Patients With Depression: A Comparison Of Frequency And Efficacy Of Psychoeducation
}

This article was published in the following Dove Press journal:

Neuropsychiatric Disease and Treatment

\author{
Tetsu Tomita' \\ Shuhei Kudo ${ }^{2}$ \\ Norio Sugawara ${ }^{3}$ \\ Akira Fujii ${ }^{4}$ \\ Koji Tsuruga $\mathbb{D}^{5}$ \\ Yasushi Sato' \\ Masamichi Ishioka (iD) \\ Kazuhiko Nakamura' \\ Norio Yasui-Furukori' \\ 'Department of Neuropsychiatry, \\ Graduate School of Medicine, Hirosaki \\ University, Hirosaki, Japan; ${ }^{2}$ Department \\ of Psychiatry, Tsugaru General Hospital, \\ Goshogawara, Japan; ${ }^{3}$ Department of \\ Clinical Epidemiology, Translational \\ Medical Center, National Center of \\ Neurology and Psychiatry, Kodaira, Japan; \\ ${ }^{4}$ Department of Mental Health, Mutsu \\ General Hospital, Mutsu, Japan; \\ ${ }^{5}$ Department of Psychiatry, Aomori \\ Prefectural Tsukushigaoka Hospital, \\ Aomori, Japan; ${ }^{6}$ Department of \\ Psychiatry, Minato Hospital, Hachinohe, \\ Japan
}

Background: We aimed to reveal sex differences in depression comprehension by reanalyzing data from a previous study of patients who were administered antidepressants.

Methods: A total of 424 outpatients were enrolled in the study. Participants were provided an original self-administered questionnaire that comprised eight items: depressive symptoms, course of depression, cause of depression, treatment plan, duration of antidepressant use, how to discontinue antidepressants, side effects of antidepressants, and psychotherapy. Each item consisted of the following two questions: "Have you received an explanation from the doctor in charge?" and "How much do you understand about your treatment?" The level of patients' comprehension of these questions was rated on a scale of 0-10 (11 anchor points). Symptoms were evaluated using the Quick Inventory for Depressive Symptomatology, Japanese version, and other scales. Patients were divided on the basis of sex, regardless of whether they were in remission.

Results: Compared with male patients, female patients with depression exhibited lower levels of depression and did not receive adequate psychoeducation from their physicians. While depression comprehension of female patients might not necessarily be associated with remission, male patients in remission received more explanations about depression and understood more compared with female patients.

Conclusion: Depression comprehension of male patients might be associated with remission, and psychoeducation should be sex-oriented to improve treatment responses.

Keywords: sex differences, psychoeducation, depression

\section{Introduction}

Patients with depression face several problems in clinical settings. First, the number of patients with depression who visit psychiatrists is very low, with approximately $18 \%$ of these patients having visited psychiatrists in Japan. ${ }^{1}$ Furthermore, after visiting psychiatrists and starting medication, it is difficult for many patients to continue the medication. Sawada et al reported that after 6 months, approximately $60 \%$ of the patients did not continue their antidepressant regimen and $63.1 \%$ of the patients did so without discussion with their physicians. ${ }^{2}$

Psychoeducation is one way to support improvement in psychiatric disorders. Department of Neuropsychiatry Graduate School of Medicine, Hirosaki University, 5 Zaifu-cho, Hirosaki 0368562, Japan

Tel +8I 172395066

$\mathrm{Fax}+81 \quad 172395067$

Email ttomita1984@yahoo.co.jp
Brown et al started the use of psychoeducational approach in the 1970s for families of patients with schizophrenia. ${ }^{3}$ Kemp et al in their study showed that better adherence and outcomes might be achieved via "compliance therapy" for patients with schizophrenia. ${ }^{4}$ Cardoso et al reported that combination therapy including 
pharmacological treatment and a brief psychoeducational approach was more effective than routine pharmacological treatment for the betterment of the quality of life of patients with bipolar disorder. ${ }^{5}$ Similarly, many reports studying psychoeducational approaches have demonstrated the importance and/or effectiveness of psychoeducation for patients with depression. For example, some studies have shown that the psychoeducational approach improves adherence to pharmacological treatment, ${ }^{6,7}$ decreases the depressive symptoms of patients, ${ }^{8}$ and prevents relapse. ${ }^{9,10}$ In a meta-analysis, Donker et al concluded that brief, passive psychoeducational interventions may improve depressive symptoms of patients. ${ }^{11}$ Brown et al reported that adherence to pharmacotherapy might be improved by providing key messages (e.g., "told what to do if there were questions," "told how long to expect to take medicine," "advised of how long side effects will last," and "given advice on managing minor side effects") about antidepressants or pharmacotherapy at baseline. ${ }^{12}$ These messages seem important to patients with depression, but the authors reported that some patients did not receive enough information. $^{12}$

Sex differences between patients with depression are very important to the epidemiology and clinical course of the disorder. Some reports have shown that female patients are more likely to have depression and recurrent depression and that they easily develop depression in response to stress. ${ }^{13-15}$ Previous studies have investigated sex differences in response to various antidepressants. Female patients respond better to selective serotonin reuptake inhibitors and citalopram, whereas male patients respond better to tricyclic antidepressants. ${ }^{16-18}$ From a cellular and molecular perspective, using positron emission tomography studies, Javanovic et al and Parsey et al showed that female patients have higher 5-HT1A receptor binding potential and lower serotonin transporter-binding potential in many brain regions than male patients. ${ }^{19,20}$ Furthermore, Bethea et al showed the influence of estrogen on serotonin transporter expression in the hypothalamus. ${ }^{21}$ Physicians may consider these sex differences when treating patients with depression.

Thus, psychoeducation is important for patients with depression, and there are sex differences in depression. Because the prevalence of psychoeducation and level of depression comprehension among patients had not been examined in detail, we evaluated depression comprehension in patients with depressive episodes or with experience of depressive episodes as well as in those who had taken or were taking antidepressants in a previous study. ${ }^{22}$
We found low levels of depression comprehension among patients, and several questionnaires that evaluated depression comprehension were found to be significantly associated with the severity of depression in the secondary results. $^{22,23}$

We have previously studied the comprehension and characteristics of patients with depression, ${ }^{22,23}$ but we did not analyze sex differences between male and female patients. In the present study, we reanalyzed data from our previous study and investigated sex differences in depression comprehension and their relationship to treatment response.

\section{Methods}

\section{Participants}

The present study was conducted from February 2013 to October 2013. Outpatients who were experiencing or had experienced a depressive episode and were taking or had taken antidepressants were recruited. Patients with mental retardation, severe depression, severe dementia, or blindness or those who did not complete the questionnaire about depression comprehension were excluded. Patients with delusion, suicidal ideation, or sub-stupor were excluded because questionnaires are often invasive and unethical for patients with such status. In total, 424 outpatients were enrolled in the study; response rate was $88.3 \%$. The participants comprised outpatients of six hospitals in Aomori and Akita, Japan: the Hirosaki University School of Medicine and Hospital, Hirosaki Aiseikai Hospital, Seihoku Chuoh Hospital, Kuroisi General Hospital, Odate City General Hospital, and Mutsu General Hospital.

The study was conducted in accordance with the Declaration of Helsinki and approved by the Ethics Committee of the Hirosaki University Hospital. All participants provided written informed consent before participation.

\section{Measures}

To assess the level of depression comprehension among the participants, we used a self-administered, original questionnaire comprising two questions "Have you received an explanation from the doctor in charge?" and "How much do you understand about your treatment?" that addressed eight categories: (A) depressive symptoms; (B) course of depression; (C) cause of depression; (D) treatment plan; (E) duration of antidepressant use; (F) how to discontinue antidepressants; $(G)$ side effects of antidepressants; and $(\mathrm{H})$ psychotherapy. The first question 
was answered with "yes" or "no" for each category. Comprehension level was rated on an 11-point scale from 0 ("I do not understand it at all") to 10 ("I understand it perfectly") for each category.

We used the Quick Inventory for Depressive Symptoms, Japanese Version (QIDS-J), to evaluate the severity of depression in each patient and defined participants with QIDS scores $\leq 5$ as remitters in the present study. Both the validity and reliability of QIDS-J have been previously established. ${ }^{24-26}$

All participants were assessed using the Clinical Global Impression-Severity (CGI-S) scales and Global Assessment of Functioning (GAF) to evaluate illness severity and general functioning. GAF is a numerical scale ( $1=$ worst to $100=$ best $)$ used to subjectively evaluate patients' psychological, occupational, and social functions. CGI-S is commonly used to measure treatment efficacy, treatment response, and symptom severity in studies of patients with mental disorders ${ }^{27}$ it uses a seven-point scale, 1 (normal, not at all ill) to 7 (extremely ill), to rate the severity of a patient's illness at the time of assessment and is based on clinician's past experience.

We collected data regarding patient demographics, diagnoses, and other clinical information from medical records. All participants were diagnosed using Diagnostic and Statistical Manual of Mental Disorders, Fourth Edition (DSM-IV).

\section{Statistical Analyses}

The characteristics of remitters and non-remitters in terms of clinical and epidemiological items were compared using chi-square tests.

The rates of remission based on receiving explanations and comprehension scores were compared between the younger and older groups using chi-square tests.

To study whether receiving explanations was associated with comprehension scores, we compared the comprehension score for each item based on whether participants had received a prior explanation about the item between the younger and older groups using $t$-tests.

We also compared the characteristics of receiving explanations and comprehension scores between remitters and non-remitters in the male and female groups using chisquare and $t$-tests.

A $P$-value $<0.05$ was considered statistically significant. Data were analyzed using SPSS for Windows 22 (IBM Japan, Tokyo, Japan).

\section{Results}

Patient Characteristics, Comparison

Between Remitters And Non-Remitters, And Comparison Between Male And

\section{Female Patients}

Table 1 shows the characteristics of participants and the results of comparisons between remitters and non-remitters and between male and female patients.

The age and GAF score of remitters were significantly higher than those of non-remitters. Conversely, the number of major depressive episodes, CGI-S scores, and QIDS-J scores of remitters were significantly lower than those of non-remitters. There was no significant difference in the contents of diagnostic criteria between remitters and nonremitters. Although the comprehension scores for items B and $\mathrm{F}$ were significantly higher in the remitters, there was no significant difference in the rate of receiving explanation regarding each item between remitters and nonremitters.

The average age, duration of disease, CGI-S, and QIDS-J were significantly lower in the male group than in the female group. GAF scores were significantly higher in the male group than in the female group. The contents of diagnostic criteria were not significantly different between the two groups. The rates of receiving explanations regarding items $\mathrm{A}, \mathrm{B}, \mathrm{C}, \mathrm{D}$, and F were significantly higher in male patients than in female patients. The comprehension scores of items B, C, D, and E, along with total comprehension scores were significantly higher in male patients than in female patients.

\section{Comparison Of Comprehension Scores In Male And Female Subjects Between Patients Receiving And Not Receiving Explanations}

Table 2 shows the comparison of comprehension scores between patients who did and did not receive explanations for each item in the male and female groups (e.g., the top of the table shows a comparison of the comprehension scores in male and female patients for item A between those who did and did not receive an explanation of item A).

Almost all comparisons showed significant differences between patients who did and did not receive explanations. In all comparisons, groups who received explanations 
Table I Characteristics Of Entire Subjects And The Comparison Between Remitters And Non-Remitters And Between Male And Female Subjects

\begin{tabular}{|c|c|c|c|c|c|c|c|c|}
\hline & & $\begin{array}{l}\text { Entire } \\
\text { Subjects } \\
(n=424)\end{array}$ & $\begin{array}{l}\text { Remitters } \\
(n=122)\end{array}$ & $\begin{array}{l}\text { Non- } \\
\text { Remitters } \\
(n=269)\end{array}$ & p-Value & Male $(n=134)$ & $\begin{array}{l}\text { Female } \\
(n=290)\end{array}$ & p-Value \\
\hline \multicolumn{2}{|c|}{$\begin{array}{l}\text { Age } \\
\text { Sex (male:female) }\end{array}$} & $56.1 \pm 16.9$ & $59.7 \pm 15.0$ & $53.2 \pm 17.3$ & $0.000^{* *}$ & $54.3 \pm 16.5$ & $56.9 \pm 17.1$ & $0.000 * *$ \\
\hline Diagnosis (n) & $\begin{array}{l}\text { Depression } \\
\text { Bipolar } \\
\text { disorder } \\
\text { Dysthymic } \\
\text { disorder } \\
\text { Personality } \\
\text { disorder } \\
\text { Others }\end{array}$ & $\begin{array}{l}364 \\
27 \\
10 \\
10 \\
13\end{array}$ & $\begin{array}{l}110 \\
8 \\
0 \\
2 \\
2\end{array}$ & $\begin{array}{l}224 \\
19 \\
10 \\
7 \\
9\end{array}$ & 0.123 & $\begin{array}{l}117 \\
10 \\
2 \\
3 \\
2\end{array}$ & $\begin{array}{l}247 \\
17 \\
8 \\
7 \\
11\end{array}$ & 0.621 \\
\hline \multicolumn{2}{|l|}{ Age at onset } & $47.6 \pm 16.9$ & $51.7 \pm 16.5$ & $45.1 \pm 16.9$ & $0.000 * *$ & $46.7 \pm 16.1$ & $48.0 \pm 17.2$ & 0.484 \\
\hline \multicolumn{2}{|c|}{ Duration of disease (years) } & $8.8 \pm 9.0$ & $8.4 \pm 8.6$ & $8.5 \pm 9.0$ & 0.964 & $7.4 \pm 7.6$ & $9.4 \pm 9.5$ & $0.028 *$ \\
\hline \multicolumn{2}{|c|}{$\begin{array}{l}\text { Duration of taking } \\
\text { antidepressants (years) }\end{array}$} & $6.9 \pm 7.2$ & $6.8 \pm 7.3$ & $6.5 \pm 6.9$ & 0.667 & $6.0 \pm 6.7$ & $7.3 \pm 7.4$ & 0.086 \\
\hline \multicolumn{2}{|c|}{$\begin{array}{l}\text { Number of major depressive } \\
\text { episodes (times) }\end{array}$} & $1.9 \pm 1.6$ & $1.6 \pm 1.4$ & $2.0 \pm 1.7$ & $0.029 *$ & $1.7 \pm 1.4$ & $1.9 \pm 1.7$ & 0.216 \\
\hline \multicolumn{2}{|c|}{$\begin{array}{l}\text { Number of hospitalizations } \\
\text { (times) }\end{array}$} & $0.4 \pm 1.1$ & $0.4 \pm 1.1$ & $0.4 \pm 1.0$ & 0.947 & $0.4 \pm 1.0$ & $0.5 \pm 1.1$ & 0.526 \\
\hline \multicolumn{2}{|l|}{ GAF } & $66.1 \pm 13.5$ & $71.4 \pm 12.2$ & $63.1 \pm 13.2$ & $0.000^{* *}$ & $68.2 \pm 12.6$ & $65.1 \pm 13.7$ & $0.027^{*}$ \\
\hline \multicolumn{2}{|l|}{ CGI-S } & $3.3 \pm 1.0$ & $2.9 \pm 0.9$ & $3.6 \pm 0.9$ & $0.000^{* *}$ & $3.1 \pm 0.9$ & $3.4 \pm 1.0$ & $0.002^{* *}$ \\
\hline \multicolumn{2}{|l|}{ QIDS-J } & $9.2 \pm 5.7$ & $2.9 \pm 1.7$ & $12.1 \pm 4.4$ & $0.000 * *$ & $8.0 \pm 5.5$ & $9.8 \pm 5.7$ & $0.003^{* *}$ \\
\hline \multicolumn{2}{|c|}{ Remitters/Non-remitters } & $122: 269$ & & & & $47: 77$ & $75: 192$ & 0.051 \\
\hline \multirow{8}{*}{$\begin{array}{l}\text { Receiving } \\
\text { explanation (yes } \\
\text { no) }\end{array}$} & A & $261: 161$ & $76: 46$ & $166: 102$ & 0.947 & $97: 37$ & $164: 124$ & $0.002 * *$ \\
\hline & B & $206: 213$ & $67: 55$ & $126: 139$ & 0.178 & $80: 52$ & $126: 161$ & $0.00 I^{* *}$ \\
\hline & C & $213: 206$ & $62: 58$ & $137: 130$ & 0.946 & $83: 51$ & $130: 155$ & $0.002^{* *}$ \\
\hline & $D$ & 239:179 & $68: 54$ & $152: 113$ & 0.765 & $91: 42$ & |48:137 & $0.002^{* *}$ \\
\hline & E & 193:224 & $54: 68$ & $|24: 14|$ & 0.643 & $69: 63$ & $|24: 16|$ & 0.095 \\
\hline & $\mathrm{F}$ & | 19:298 & $35: 87$ & $75: 191$ & 0.920 & $47: 85$ & $72: 213$ & $0.030 *$ \\
\hline & G & $211: 206$ & $58: 63$ & $143: 124$ & 0.304 & 73.58 & |38:148 & 0.157 \\
\hline & $\mathrm{H}$ & $150: 265$ & $42: 79$ & I0I:165 & 0.538 & $52: 81$ & $98: 184$ & 0.390 \\
\hline \multirow{9}{*}{$\begin{array}{l}\text { Comprehension } \\
\text { scores }\end{array}$} & A & $5.2 \pm 2.8$ & $5.4 \pm 2.9$ & $5.1 \pm 2.8$ & 0.391 & $5.6 \pm 2.8$ & $5.1 \pm 2.8$ & 0.077 \\
\hline & B & $4.6 \pm 2.9$ & $5.1 \pm 3.1$ & $4.4 \pm 2.8$ & $0.026^{*}$ & $5.4 \pm 2.9$ & $4.3 \pm 2.9$ & $0.000^{* *}$ \\
\hline & C & $5.0 \pm 3.0$ & $5.0 \pm 3.2$ & $5.0 \pm 2.9$ & 0.917 & $5.5 \pm 3.0$ & $4.8 \pm 3.0$ & $0.026^{*}$ \\
\hline & $D$ & $4.7 \pm 3.0$ & $5.0 \pm 3.1$ & $4.6 \pm 3.0$ & 0.216 & $5.5 \pm 3.0$ & $4.3 \pm 3.0$ & $0.000 * *$ \\
\hline & $E$ & $3.9 \pm 3.1$ & $4.3 \pm 3.3$ & $3.7 \pm 3.0$ & 0.073 & $4.4 \pm 3.1$ & $3.7 \pm 3.1$ & $0.025^{*}$ \\
\hline & $\mathrm{F}$ & $3.2 \pm 3.0$ & $3.7 \pm 3.3$ & $2.8 \pm 2.8$ & $0.009 * *$ & $3.5 \pm 3.1$ & $3.1 \pm 3.0$ & 0.216 \\
\hline & G & $3.9 \pm 3.1$ & $4.4 \pm 3.3$ & $3.7 \pm 3.0$ & 0.056 & $4.2 \pm 3.2$ & $3.8 \pm 3.1$ & 0.227 \\
\hline & $\mathrm{H}$ & $3.3 \pm 3.0$ & $3.7 \pm 3.2$ & $3.1 \pm 2.9$ & 0.050 & $3.7 \pm 3.1$ & $3.1 \pm 2.9$ & 0.103 \\
\hline & Total & $33.8 \pm 20.2$ & $35.8 \pm 22.2$ & $32.7 \pm 19.1$ & 0.251 & $37.2 \pm 20.2$ & $32.1 \pm 20.0$ & $0.003^{* *}$ \\
\hline
\end{tabular}

Notes: ${ }^{*} \mathrm{p}<0.05,{ }^{* *} \mathrm{p}<0.01$. A: depressive symptoms, B: the course of depression, C: the cause of depression, D: the treatment plan, E: the duration of antidepressant uses, F: how to discontinue antidepressant, G: the side effects of antidepressants, H: psychotherapy.

Abbreviation: GAF, The Global Assessment of Functioning; CGI-S, The Clinical Global Impression-Severity scale; QIDS-J, Quick Inventory of Depressive Symptomatology Japanese version. 
Table 2 Comparison For Both Male And Female Subjects Of The Comprehension Scores Between Patients Divided By Whether They Received Explanations

\begin{tabular}{|c|c|c|c|c|c|c|c|}
\hline \multirow[t]{2}{*}{ Receiving Explanation } & \multirow[t]{2}{*}{ Comprehension Scores } & \multicolumn{3}{|l|}{ Male } & \multicolumn{3}{|l|}{ Female } \\
\hline & & Received & Not Received & p-Value & Received & Not Received & p-Value \\
\hline \multirow[t]{9}{*}{ Item A } & $A$ & $6.5 \pm 2.4$ & $3.3 \pm 2.7$ & 0.000 & $6.0 \pm 2.4$ & $3.8 \pm 2.8$ & 0.000 \\
\hline & B & $6.3 \pm 2.4$ & $3.0 \pm 2.7$ & 0.000 & $5.2 \pm 2.7$ & $3.1 \pm 2.7$ & 0.000 \\
\hline & $\mathrm{C}$ & $6.6 \pm 2.4$ & $2.8 \pm 2.7$ & 0.000 & $5.7 \pm 2.7$ & $3.6 \pm 3.1$ & 0.000 \\
\hline & $\mathrm{D}$ & $6.5 \pm 2.4$ & $3.1 \pm 3.0$ & 0.000 & $5.3 \pm 2.7$ & $3.0 \pm 2.7$ & 0.000 \\
\hline & $\mathrm{E}$ & $5.2 \pm 2.9$ & $2.4 \pm 2.8$ & 0.000 & $4.6 \pm 2.9$ & $2.5 \pm 2.9$ & 0.000 \\
\hline & $\mathrm{F}$ & $4.3 \pm 3.1$ & $1.3 \pm 1.7$ & 0.000 & $3.7 \pm 3.0$ & $2.2 \pm 2.7$ & 0.000 \\
\hline & G & $5.1 \pm 3.2$ & $2.0 \pm 2.2$ & 0.000 & $4.6 \pm 3.1$ & $2.7 \pm 2.8$ & 0.000 \\
\hline & $\mathrm{H}$ & $4.4 \pm 3.0$ & $1.7 \pm 2.3$ & 0.000 & $4.0 \pm 3.0$ & $2.0 \pm 2.4$ & 0.000 \\
\hline & Total & $44.3 \pm 17.1$ & $19.5 \pm 16.3$ & 0.000 & $39.5 \pm 17.9$ & $21.7 \pm 18.0$ & 0.000 \\
\hline \multirow[t]{9}{*}{ Item B } & $A$ & $6.4 \pm 2.4$ & $4.2 \pm 3.0$ & 0.000 & $6.2 \pm 2.3$ & $4.1 \pm 2.8$ & 0.000 \\
\hline & B & $6.5 \pm 2.2$ & $3.6 \pm 2.9$ & 0.000 & $5.7 \pm 2.5$ & $3.2 \pm 2.7$ & 0.000 \\
\hline & C & $6.7 \pm 2.2$ & $3.7 \pm 3.3$ & 0.000 & $5.9 \pm 2.5$ & $3.9 \pm 3.1$ & 0.000 \\
\hline & $D$ & $6.7 \pm 2.1$ & $3.7 \pm 3.1$ & 0.000 & $5.8 \pm 2.4$ & $3.2 \pm 2.8$ & 0.000 \\
\hline & $E$ & $5.5 \pm 2.8$ & $2.8 \pm 2.8$ & 0.000 & $5.0 \pm 2.9$ & $2.7 \pm 2.9$ & 0.000 \\
\hline & $\mathrm{F}$ & $4.3 \pm 3.0$ & $2.0 \pm 2.6$ & 0.000 & $3.9 \pm 3.1$ & $2.4 \pm 2.7$ & 0.000 \\
\hline & G & $4.9 \pm 3.1$ & $3.0 \pm 3.1$ & 0.000 & $4.8 \pm 3.1$ & $3.0 \pm 2.8$ & 0.000 \\
\hline & $\mathrm{H}$ & $4.7 \pm 3.0$ & $2.0 \pm 2.4$ & 0.000 & $4.1 \pm 2.9$ & $2.4 \pm 2.7$ & 0.000 \\
\hline & Total & $45.4 \pm 16.6$ & $25.0 \pm 19.0$ & 0.000 & $41.9 \pm 16.3$ & $23.8 \pm 18.8$ & 0.000 \\
\hline \multirow[t]{9}{*}{ Item C } & $A$ & $6.6 \pm 2.5$ & $3.9 \pm 2.7$ & 0.000 & $6.3 \pm 2.2$ & $4.0 \pm 2.8$ & 0.000 \\
\hline & B & $6.4 \pm 2.4$ & $3.7 \pm 2.9$ & 0.000 & $5.5 \pm 2.5$ & $3.2 \pm 2.7$ & 0.000 \\
\hline & C & $6.8 \pm 2.3$ & $3.4 \pm 2.8$ & 0.000 & $6.2 \pm 2.0$ & $3.6 \pm 3.2$ & 0.000 \\
\hline & $D$ & $6.6 \pm 2.2$ & $3.7 \pm 3.2$ & 0.000 & $5.7 \pm 2.5$ & $3.1 \pm 2.9$ & 0.000 \\
\hline & $E$ & $5.3 \pm 3.0$ & $3.0 \pm 2.7$ & 0.000 & $4.9 \pm 2.7$ & $2.7 \pm 3.1$ & 0.000 \\
\hline & $\mathrm{F}$ & $4.2 \pm 3.2$ & $2.2 \pm 2.5$ & 0.000 & $3.9 \pm 2.9$ & $2.3 \pm 2.8$ & 0.000 \\
\hline & G & $5.2 \pm 3.1$ & $2.6 \pm 2.7$ & 0.000 & $4.9 \pm 3.0$ & $2.9 \pm 2.9$ & 0.000 \\
\hline & $\mathrm{H}$ & $4.5 \pm 3.1$ & $2.2 \pm 2.6$ & 0.000 & $4.1 \pm 2.9$ & $2.3 \pm 2.7$ & 0.000 \\
\hline & Total & $45.2 \pm 17.0$ & $24.8 \pm 18.6$ & 0.000 & $42.0 \pm 16.0$ & $23.4 \pm 18.9$ & 0.000 \\
\hline \multirow[t]{9}{*}{ Item D } & $A$ & $6.4 \pm 2.4$ & $3.7 \pm 2.9$ & 0.000 & $5.9 \pm 2.4$ & $4.1 \pm 2.9$ & 0.000 \\
\hline & B & $6.4 \pm 2.3$ & $3.2 \pm 2.7$ & 0.000 & $5.3 \pm 2.6$ & $3.1 \pm 2.8$ & 0.000 \\
\hline & C & $6.6 \pm 2.3$ & $3.2 \pm 3.0$ & 0.000 & $5.9 \pm 2.5$ & $3.7 \pm 3.2$ & 0.000 \\
\hline & $\mathrm{D}$ & $6.7 \pm 2.1$ & $2.9 \pm 2.9$ & 0.000 & $5.7 \pm 2.4$ & $2.8 \pm 2.8$ & 0.000 \\
\hline & $E$ & $5.4 \pm 2.9$ & $2.4 \pm 2.5$ & 0.000 & $5.0 \pm 2.8$ & $2.3 \pm 2.8$ & 0.000 \\
\hline & $\mathrm{F}$ & $4.3 \pm 3.1$ & $1.5 \pm 1.9$ & 0.000 & $3.9 \pm 2.9$ & $2.1 \pm 2.8$ & 0.000 \\
\hline & G & $5.0 \pm 3.1$ & $2.3 \pm 2.4$ & 0.000 & $4.9 \pm 2.8$ & $2.7 \pm 2.9$ & 0.000 \\
\hline & $\mathrm{H}$ & $4.5 \pm 3.1$ & $1.7 \pm 2.1$ & 0.000 & $4.2 \pm 2.8$ & $2.1 \pm 2.7$ & 0.000 \\
\hline & Total & $45.1 \pm 16.8$ & $20.9 \pm 16.5$ & 0.000 & $41.3 \pm 16.8$ & $22.0 \pm 18.4$ & 0.000 \\
\hline \multirow[t]{9}{*}{ Item E } & A & $6.5 \pm 2.3$ & $4.6 \pm 3.1$ & 0.000 & $6.0 \pm 2.4$ & $4.3 \pm 2.8$ & 0.000 \\
\hline & B & $6.6 \pm 2.3$ & $4.1 \pm 3.0$ & 0.000 & $5.6 \pm 2.5$ & $3.3 \pm 2.8$ & 0.000 \\
\hline & C & $6.7 \pm 2.1$ & $4.3 \pm 3.3$ & 0.000 & $6.1 \pm 2.5$ & $3.8 \pm 3.1$ & 0.000 \\
\hline & $\mathrm{D}$ & $6.8 \pm 2.0$ & $4.2 \pm 3.2$ & 0.000 & $6.0 \pm 2.3$ & $3.0 \pm 2.8$ & 0.000 \\
\hline & $E$ & $6.3 \pm 2.2$ & $2.4 \pm 2.7$ & 0.000 & $5.8 \pm 2.6$ & $2.1 \pm 2.5$ & 0.000 \\
\hline & $\mathrm{F}$ & $4.7 \pm 2.9$ & $2.0 \pm 2.6$ & 0.000 & 4. $1 \pm 2.9$ & $2.3 \pm 2.8$ & 0.000 \\
\hline & G & $5.2 \pm 3.1$ & $3.1 \pm 2.9$ & 0.000 & $4.9 \pm 2.9$ & $3.0 \pm 2.9$ & 0.000 \\
\hline & $\mathrm{H}$ & $4.9 \pm 3.1$ & $2.3 \pm 2.5$ & 0.000 & $4.3 \pm 2.8$ & $2.3 \pm 2.7$ & 0.000 \\
\hline & Total & $47.4 \pm 15.9$ & $26.8 \pm 18.8$ & 0.000 & $43.1 \pm 16.9$ & $23.3 \pm 17.9$ & 0.000 \\
\hline Item F & A & $6.4 \pm 2.4$ & $5.1 \pm 3.0$ & 0.011 & $6.6 \pm 2.1$ & $4.5 \pm 2.8$ & 0.000 \\
\hline
\end{tabular}

(Continued) 
Table 2 (Continued).

\begin{tabular}{|c|c|c|c|c|c|c|c|}
\hline \multirow[t]{2}{*}{ Receiving Explanation } & \multirow[t]{2}{*}{ Comprehension Scores } & \multicolumn{3}{|l|}{ Male } & \multicolumn{3}{|l|}{ Female } \\
\hline & & Received & Not Received & p-Value & Received & Not Received & p-Value \\
\hline & $\begin{array}{l}\text { B } \\
\text { C } \\
\text { D } \\
\text { E } \\
\text { F } \\
\text { G } \\
\text { H } \\
\text { Total }\end{array}$ & $\begin{array}{l}6.4 \pm 2.4 \\
6.4 \pm 2.4 \\
6.6 \pm 2.1 \\
6.2 \pm 2.4 \\
5.6 \pm 2.5 \\
5.1 \pm 3.0 \\
4.9 \pm 2.9 \\
47.4 \pm 16.6\end{array}$ & $\begin{array}{l}4.9 \pm 3.0 \\
5.1 \pm 3.2 \\
4.9 \pm 3.2 \\
3.5 \pm 3.0 \\
2.2 \pm 2.6 \\
3.7 \pm 3.2 \\
3.0 \pm 3.0 \\
32.0 \pm 20.0\end{array}$ & $\begin{array}{l}0.004 \\
0.026 \\
0.000 \\
0.000 \\
0.000 \\
0.013 \\
0.001 \\
0.000\end{array}$ & $\begin{array}{l}6.0 \pm 2.1 \\
6.3 \pm 2.3 \\
5.9 \pm 2.5 \\
5.6 \pm 2.4 \\
6.0 \pm 2.1 \\
5.9 \pm 2.5 \\
4.9 \pm 2.6 \\
47.3 \pm 14.3\end{array}$ & $\begin{array}{l}3.6 \pm 2.8 \\
4.3 \pm 3.1 \\
3.7 \pm 2.9 \\
3.0 \pm 3.1 \\
2.0 \pm 2.5 \\
3.0 \pm 2.9 \\
2.5 \pm 2.7 \\
26.0 \pm 18.5\end{array}$ & $\begin{array}{l}0.000 \\
0.000 \\
0.000 \\
0.000 \\
0.000 \\
0.000 \\
0.000 \\
0.000\end{array}$ \\
\hline Item G & $\begin{array}{l}\text { A } \\
\text { B } \\
\text { C } \\
\text { D } \\
\text { E } \\
\text { F } \\
\text { G } \\
\text { H } \\
\text { Total }\end{array}$ & $\begin{array}{l}6.6 \pm 2.4 \\
6.4 \pm 2.4 \\
6.8 \pm 2.4 \\
6.8 \pm 2.2 \\
5.5 \pm 3.0 \\
4.3 \pm 3.1 \\
5.7 \pm 2.8 \\
4.6 \pm 3.1 \\
46.6 \pm 16.7\end{array}$ & $\begin{array}{l}4.4 \pm 2.9 \\
4.1 \pm 2.9 \\
4.1 \pm 3.0 \\
4.0 \pm 3.1 \\
3.1 \pm 2.8 \\
2.3 \pm 2.6 \\
2.2 \pm 2.5 \\
2.5 \pm 2.7 \\
25.8 \pm 18.5\end{array}$ & $\begin{array}{l}0.000 \\
0.000 \\
0.000 \\
0.000 \\
0.000 \\
0.000 \\
0.000 \\
0.000 \\
0.000\end{array}$ & $\begin{array}{l}6.2 \pm 2.3 \\
5.4 \pm 2.7 \\
6.0 \pm 2.5 \\
5.5 \pm 2.7 \\
4.7 \pm 3.0 \\
4.1 \pm 3.0 \\
5.7 \pm 2.3 \\
4.5 \pm 2.8 \\
42.1 \pm 16.8\end{array}$ & $\begin{array}{l}4.0 \pm 2.8 \\
3.2 \pm 2.7 \\
3.7 \pm 3.0 \\
3.2 \pm 2.8 \\
2.8 \pm 2.9 \\
2.1 \pm 2.7 \\
2.0 \pm 2.5 \\
1.9 \pm 2.4 \\
22.0 \pm 17.8\end{array}$ & $\begin{array}{l}0.000 \\
0.000 \\
0.000 \\
0.000 \\
0.000 \\
0.000 \\
0.000 \\
0.000 \\
0.000\end{array}$ \\
\hline Item $\mathrm{H}$ & $\begin{array}{l}\text { A } \\
\text { B } \\
\text { C } \\
\text { D } \\
\text { E } \\
\text { F } \\
\text { G } \\
\text { H } \\
\text { Total }\end{array}$ & $\begin{array}{l}6.2 \pm 2.6 \\
6.2 \pm 2.5 \\
6.6 \pm 2.5 \\
6.3 \pm 2.3 \\
5.6 \pm 2.9 \\
4.4 \pm 3.0 \\
4.9 \pm 3.0 \\
5.8 \pm 2.5 \\
45.5 \pm 17.8\end{array}$ & $\begin{array}{l}5.2 \pm 3.0 \\
4.9 \pm 3.1 \\
4.9 \pm 3.1 \\
5.0 \pm 3.2 \\
3.7 \pm 3.0 \\
2.8 \pm 3.0 \\
3.8 \pm 3.3 \\
2.3 \pm 2.6 \\
32.0 \pm 19.9\end{array}$ & $\begin{array}{l}0.053 \\
0.013 \\
0.002 \\
0.006 \\
0.001 \\
0.003 \\
0.038 \\
0.000 \\
0.000\end{array}$ & $\begin{array}{l}6.2 \pm 2.2 \\
5.8 \pm 2.6 \\
6.2 \pm 2.6 \\
5.8 \pm 2.5 \\
5.2 \pm 2.9 \\
4.4 \pm 2.9 \\
5.5 \pm 2.7 \\
5.3 \pm 2.3 \\
44.6 \pm 16.3\end{array}$ & $\begin{array}{l}4.4 \pm 2.9 \\
3.4 \pm 2.8 \\
4.1 \pm 3.1 \\
3.5 \pm 2.9 \\
2.9 \pm 3.0 \\
2.3 \pm 2.8 \\
2.9 \pm 2.9 \\
1.9 \pm 2.5 \\
24.6 \pm 18.3\end{array}$ & $\begin{array}{l}0.000 \\
0.000 \\
0.000 \\
0.000 \\
0.000 \\
0.000 \\
0.000 \\
0.000 \\
0.000\end{array}$ \\
\hline
\end{tabular}

Notes: A, depressive symptoms; B, the course of depression; C, the cause of depression; $D$, the treatment plan; $E$, the duration of antidepressant uses; $F$, how to discontinue antidepressant; $G$, the side effects of antidepressants; H, psychotherapy.

exhibited higher comprehension scores for every item in both males and females.

\section{Comparison Between Remitters And Non-Remitters In Male And Female}

\section{Patients}

Table 3 shows the results of comparison between remitters and non-remitters in male and female patients. In the male group, the average age, duration of disease, duration of taking antidepressants, and GAF scores of remitters were significantly higher than those of non-remitters. In the female group, the average age, age at onset, and GAF scores of remitters were significantly higher than those of non-remitters. CGI-S and QIDS-J scores of remitters were significantly lower than those of non-remitters in both male and female patients.
None of the items regarding receiving explanation showed a significant difference between remitters and non-remitters in males or females. In the male group, the comprehension scores of remitters for items B, E, F, and $\mathrm{H}$, along with their total comprehension scores, were significantly higher than those of non-remitters. Conversely, none of the comprehension scores for individual items or for total scores showed a significant difference between remitters and non-remitters in the female group.

\section{Discussion}

In the present study, we reanalyzed data from a previous study ${ }^{22,23}$ to investigate the difference between male and female patients in terms of depression comprehension and showed the effect of psychoeducation. Compared with male patients, female patients with depression showed lower levels of depression comprehension and did not receive 
Table 3 Comparison Between Remitters And Non-Remitters For Both Male And Female Patients

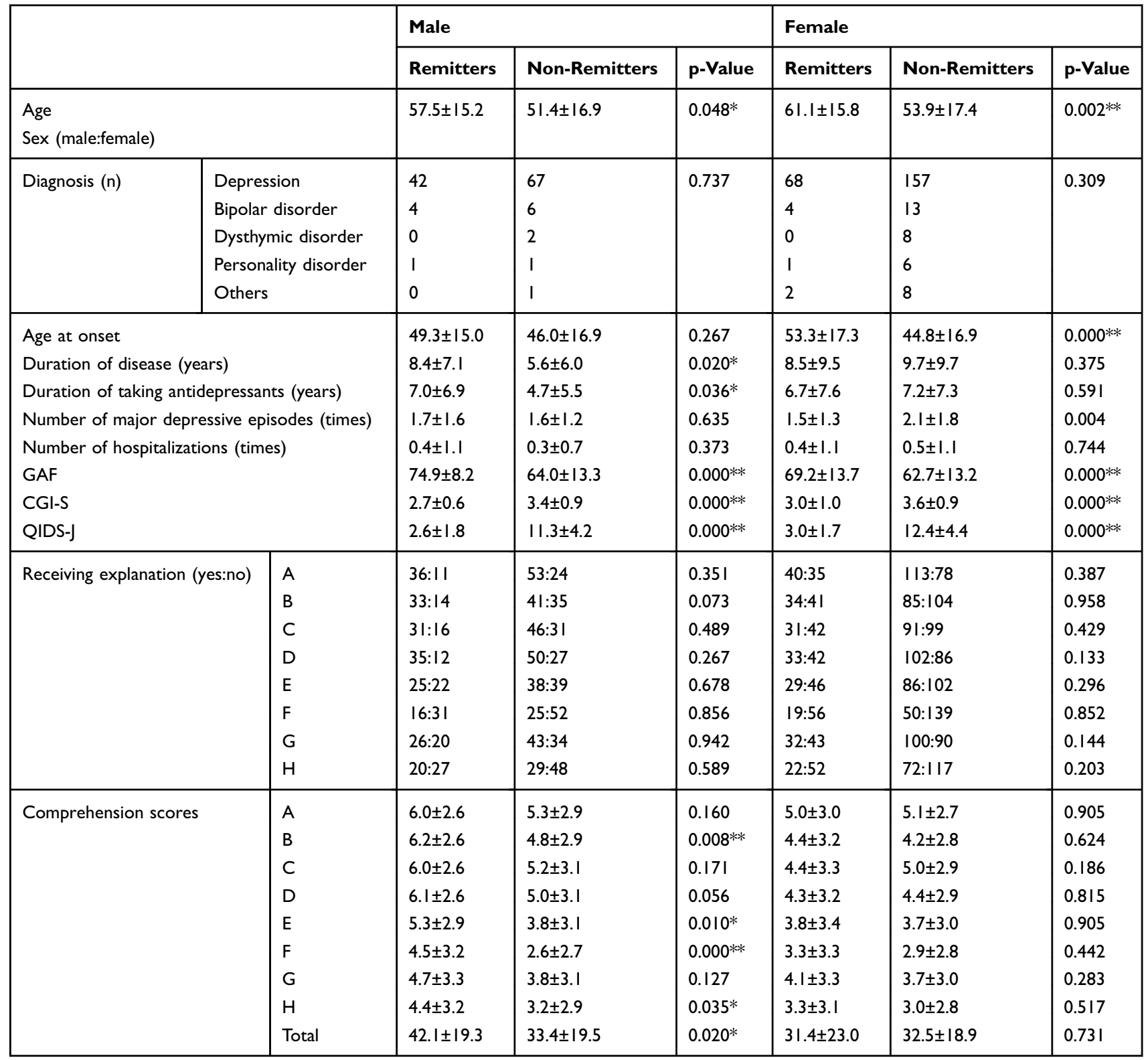

Notes: ${ }^{*}<<0.05,{ }^{* *} p<0.01$. A: depressive symptoms, B: the course of depression, $\mathrm{C}$ : the cause of depression, D: the treatment plan, E: the duration of antidepressant uses, F: how to discontinue antidepressant, G: the side effects of antidepressants, H: psychotherapy.

Abbreviations: GAF, The Global Assessment of Functioning; CGI-S, The Clinical Global Impression-severity scale; QIDS-J, Quick Inventory of Depressive Symptomatology Japanese Version.

adequate psychoeducation from their physicians in the typical treatment course. While their comprehension of depression might not necessarily be associated with remission, male patients in remission received more explanations about depression and understood more compared with female patients. To the best of our knowledge, the present study is the first to analyze differences in depression comprehension between male and female patients with depression and to study the association between such comprehension and responsiveness to depression treatment in these patients.
As indicated by the results in Table 2, both male and female patients with depression who received explanations about depression showed better depression comprehension, as evaluated using questionnaires. However, the results from Table 3 show that female patients with depression in remission did not exhibit better depression comprehension, whereas male patients with depression in remission did. The remission of female patients with depression might not be influenced by depression comprehension, which was evaluated using questionnaires including items A through to $\mathrm{H}$. 
Although the results seemingly demonstrate that psychoeducation is not useful for female patients with depression, "psychoeducation" in the present study was defined only by explanations or information. Psychoeducation is complex and includes comprehensive intervention; thus, other types of questionnaires may be preferable in future studies. In addition to explanations and information, other psychoeducational factors might positively influence the response of female patients to depression treatment.

In the present study, it was unclear why there were differences in characteristics associated with depression comprehension and remission between male and female patients. As mentioned above, certain differences in responsiveness to antidepressants and in biological characteristics might be associated with the outcomes. ${ }^{16-20}$ Krivoy et al reported that male patients with depression show better adherence to antidepressants than female patients. ${ }^{28}$ The male participants in the present study might have increased their adherence to antidepressants as a result of receiving explanations and depression comprehension, and this might have led to improved treatment responses. However, some studies have shown lower adherence in male patients and have reported that sex differences regarding better adherence as a result of receiving explanations and depression comprehension may not improve the responses. ${ }^{29,30}$ The present study was done in Japan and cultural-specific aspects (e.g., how people do when they are in trouble or whether they discuss about their mental health with others) might influence the sex differences but we did not research such aspects and influence. Different results might be reported in the study in other countries or regions.

Our results suggest that male patients with depression should receive more psychoeducation because receiving explanations and depression comprehension are associated with better treatment responses. However, we assert that it is still necessary to provide explanations to female patients and help them understand depression better. Female patients might require more psychological or emotional discussion rather than explanations during their interview and need other information besides the items from the present study to improve their treatment responses; acceptance, empathizing, supportive psychotherapy, and concrete methods to solve their troubles. In a previous study, Kornstein et al reported that female patients with among patients with chronic depression, female patients receive psychotherapy more than male patients. ${ }^{17}$ Psychiatrists might feel that female patients need psychological approaches more than male patients.

There were several limitations to the present study. First, we only used subjective evaluations of receiving explanations and comprehension of items of psychoeducation. Furthermore, "psychoeducation" in the present study was defined only on the basis of explanation and information about depression and its treatment. The results may not reflect clinical reality in terms of providing and receiving explanations and comprehension of depression and its treatment. Sleath and Rubin reported that female physicians are more likely to counsel than male physicians in the study including patients and resident physicians. ${ }^{31}$ Although we did not record the genders of physicians, the genders might influence comprehension of depression. In future assessments, we may use objective rather than subjective questionnaires and record the genders of physicians. Other types of questionnaires may be better because psychoeducation is complex and is a form of comprehensive intervention. Second, this is a retrospective study. We did not discuss when and how patients received explanations. Some patients might have received explanations via videos, booklets, and books, whereas others might only have received explanations through lectures from physicians. The timing and methods of explanations might influence the efficacy of psychoeducation. The methods of explanations should be adjusted according to a patient's severity of depression and comprehension. Intervention studies are needed to investigate the efficacious timing for explanations and to identify methods or contents that are helpful for these patients. Such studies might reveal methods for improving psychoeducation in female patients.

In conclusion, we reanalyzed data from a previous study to compare male and female patients in terms of depression comprehension and demonstrated the effect of psychoeducation. Compared with male patients, female patients with depression showed lower levels of depression comprehension and did not receive adequate psychoeducation from their physicians, but their depression comprehension might not be associated with remission. The depression comprehension of male patients, however, might be associated with remission. There may be a necessity for sex-oriented psychoeducation and related items for each patient.

\section{Acknowledgment}

The authors would like to thank all the co-workers in this study for their helpful contributions in collecting and managing the data.

\section{Disclosure}

Norio Yasui-Furukori has received grant/research support or honoraria from and has been a speaker for Astra, 
Dainippon, Eli Lilly, GSK, Janssen-Pharma, Meiji, Mochida Pharmaceutical, MSD, Otsuka Pharmaceutical, Pfizer, Takata, and Yoshitomi. Dr Norio Yasui-Furukori also reports personal fees from MSD, Otsuka Pharmaceutical, Mochida Pharmaceutical, DainipponSumitomo Pharma, outside the submitted work. The authors report no other conflicts of interest in this work.

\section{References}

1. Kawakami N, Takeshima T, Ono Y, et al. Twelve-month prevalence, severity, and treatment of common mental disorders in communities in Japan: preliminary finding from the World Mental Health Japan Survey 2002-2003. Psychiatry Clin Neurosci. 2005;59(4):441-452. doi:10.1111/pen.2005.59.issue-4

2. Sawada N, Uchida H, Suzuki T, et al. Persistence and compliance to antidepressant treatment in patients with depression: a chart review. BMC Psychiatry. 2009;9:38. doi:10.1186/1471-244X-9-38

3. Brown GW, Birley JL, Wing JK. Influence of family life on the course of schizophrenic disorders: a replication. Br J Psychiatry. 1972;121 (562):241-258. doi:10.1192/bjp.121.3.241

4. Kemp R, Hayward P, Applewhaite G, Everitt B, David A. Compliance therapy in psychotic patients: randomised controlled trial. BMJ. 1996;312(7027):345-349. doi:10.1136/bmj.312.7027.345

5. de Azevedo Cardoso T, de Azambuja Farias C, Mondin TC, et al. Brief psychoeducation for bipolar disorder: impact on quality of life in young adults in a 6-month follow-up of a randomized controlled trial. Psychiatry Res. 2014;220(3):896-902. doi:10.1016/j.psychres.2014.09.013

6. Wells KB, Sherbourne C, Schoenbaum M, et al. Impact of disseminating quality improvement programs for depression in managed primary care: a randomized controlled trial. JAMA. 2000;283 (2):212-220. doi:10.1001/jama.283.2.212

7. Rost K, Nutting P, Smith J, Werner J, Duan N. Improving depression outcomes in community primary care practice: a randomized trial of the quEST intervention. Quality Enhancement by Strategic Teaming. J Gen Intern Med. 2001;16(3):143-149. doi:10.1111/j.1525-1497.20 01.00537.x

8. Morokuma I, Shimodera S, Fujita H, et al. Psychoeducation for major depressive disorders: a randomised controlled trial. Psychiatry Res. 2013;210(1):134-139. doi:10.1016/j.psychres.2013.05.018

9. Shimazu K, Shimodera S, Mino Y, et al. Family psychoeducation for major depression: randomised controlled trial. $\mathrm{Br} J$ Psychiatry. 2011;198(5):385-390. doi:10.1192/bjp.bp.110.078626

10. Kronmuller KT, Victor D, Schenkenbach C, et al. Knowledge about affective disorders and outcome of depression. J Affect Disord. 2007;104(1-3):155-160. doi:10.1016/j.jad.2007.02.010

11. Donker T, Griffiths KM, Cuijpers P, Christensen H. Psychoeducation for depression, anxiety and psychological distress: a meta-analysis. BMC Med. 2009;7:79. doi:10.1186/1741-7015-7-79

12. Brown C, Battista DR, Sereika SM, Bruehlman RD, Dunbar-Jacob J, Thase ME. How can you improve antidepressant adherence? J Fam Pract. 2007;56(5):356-363.

13. Ernst C, Angst J. The Zurich Study. XII. Sex differences in depression. Evidence from longitudinal epidemiological data. Eur Arch Psychiatry Clin Neurosci. 1992;241(4):222-230. doi:10.1007/BF02190257

14. Kendler KS, Thornton LM, Gardner CO. Stressful life events and previous episodes in the etiology of major depression in women: an evaluation of the "kindling" hypothesis. Am J Psychiatry. 2000;157 (8):1243-1251. doi:10.1176/appi.ajp.157.8.1243

15. Kessler RC, McGonagle KA, Swartz M, Blazer DG, Nelson CB. Sex and depression in the National Comorbidity Survey. I: lifetime prevalence, chronicity and recurrence. J Affect Disord. 1993;29(2-3):85-96. doi:10.1016/0165-0327(93)90026-G
16. Khan A, Brodhead AE, Schwartz KA, Kolts RL, Brown WA. Sex differences in antidepressant response in recent antidepressant clinical trials. J Clin Psychopharmacol. 2005;25(4):318-324. doi:10.109 7/01.jcp.0000168879.03169.ce

17. Kornstein SG, Schatzberg AF, Thase ME, et al. Gender differences in chronic major and double depression. $J$ Affect Disord. 2000;60(1):111. doi:10.1016/S0165-0327(99)00158-5

18. Young EA, Kornstein SG, Marcus SM, et al. Sex differences in response to citalopram: a STAR*D report. J Psychiatr Res. 2009;43 (5):503-511. doi:10.1016/j.jpsychires.2008.07.002

19. Jovanovic H, Lundberg J, Karlsson P, et al. Sex differences in the serotonin $1 \mathrm{~A}$ receptor and serotonin transporter binding in the human brain measured by PET. Neuroimage. 2008;39(3):1408-1419. doi:10.1016/j.neuroimage.2007.10.016

20. Parsey RV, Oquendo MA, Simpson NR, et al. Effects of sex, age, and aggressive traits in man on brain serotonin 5-HT1A receptor binding potential measured by PET using [C-11]WAY-100635. Brain Res. 2002;954(2):173-182. doi:10.1016/S0006-8993(02)03243-2

21. Bethea CL, Mirkes SJ, Su A, Michelson D. Effects of oral estrogen, raloxifene and arzoxifene on gene expression in serotonin neurons of macaques. Psychoneuroendocrinology. 2002;27(4):431-445. doi:10.101 6/S0306-4530(01)00054-3

22. Kudo S, Tomita T, Sugawara N, et al. The low level of understanding of depression among patients treated with antidepressants: a survey of 424 outpatients in Japan. Neuropsychiatr Dis Treat. 2015;11:28112816. doi:10.2147/NDT.S93657

23. Tomita T, Yasui-Furukori N, Kudo S, et al. The association between the severity and level of understanding of depression among patients treated with antidepressants: a survey of 424 outpatients in Japan. Clin Neuropharmacol. 2018;41:1-5. doi:10.1097/WNF.0000000000000257

24. Rush AJ, Trivedi MH, Ibrahim HM, et al. The 16-Item Quick Inventory of Depressive Symptomatology (QIDS), clinician rating (QIDS-C), and self-report (QIDS-SR): a psychometric evaluation in patients with chronic major depression. Biol Psychiatry. 2003;54 (5):573-583. doi:10.1016/S0006-3223(02)01866-8

25. Trivedi MH, Rush AJ, Ibrahim HM, et al. The Inventory of Depressive Symptomatology, Clinician Rating (IDS-C) and Self-Report (IDS$\mathrm{SR}$ ), and the Quick Inventory of Depressive Symptomatology, Clinician Rating (QIDS-C) and Self-Report (QIDS-SR) in public sector patients with mood disorders: a psychometric evaluation. Psychol Med. 2004;34(1):73-82. doi:10.1017/s0033291703001107

26. Fujisawa D, Nakagawa A, Tajima M, et al. Reliability and validity of quick inventory of depressive symptomatology Japanese version. Seishin Shinkeigaku Zasshi. 2008;(Supplement):S-324.

27. Guy W. Clinical Global Impression Scale (CGI). ECDEU Assessment Manual for Psychopharmacology. Rockville: US Dept. of Health, Education, and Welfare, Public Health Service, Alcohol, Drug Abuse, and Mental Health Administration, National Institute of Mental Health, Psychopharmacology Research Branch, Division of Extramural Research Programs; 1976:217-222.

28. Krivoy A, Balicer RD, Feldman B, et al. The impact of age and gender on adherence to antidepressants: a 4-year population-based cohort study. Psychopharmacology (Berl). 2015;232(18):3385-3390. doi:10.1007/s00213-015-3988-9

29. Degli Esposti L, Piccinni C, Sangiorgi D, Fagiolini A, Buda S. Patterns of antidepressant use in Italy: therapy duration, adherence and switching. Clin Drug Investig. 2015;35(11):735-742. doi:10.1007/s40261015-0332-4

30. Serna MC, Real J, Cruz I, Galvan L, Martin E. Monitoring patients on chronic treatment with antidepressants between 2003 and 2011: analysis of factors associated with compliance. BMC Public Health. 2015;15:1184. doi:10.1186/s12889-015-2493-8

31. Sleath B, Rubin RH. Gender, ethnicity, and physician-patient communication about depression and anxiety in primary care. Patient Educ Couns. 2002;48(3):243-252. doi:10.1016/S0738-3991(02)001 77-5 


\section{Publish your work in this journal}

Neuropsychiatric Disease and Treatment is an international, peerreviewed journal of clinical therapeutics and pharmacology focusing on concise rapid reporting of clinical or pre-clinical studies on a range of neuropsychiatric and neurological disorders. This journal is indexed on PubMed Central, the 'PsycINFO' database and CAS, and is the official journal of The International Neuropsychiatric Association (INA). The manuscript management system is completely online and includes a very quick and fair peer-review system, which is all easy to use. Visit http://www.dovepress.com/testimonials.php to read real quotes from published authors. 